\title{
A Basic and Clinical Perspective on the Future of Perioperative Therapy for Gastric Cancer
}

\author{
Jiang Chen ${ }^{1,2, *}$, Dan G. Duda ${ }^{1}$, Aya Matsui ${ }^{1,3}$, Hiroto Kikuchi ${ }^{1,4}$, Kazumichi Kawakubo ${ }^{1}$, Shuji Kitahara ${ }^{3, *}$ \\ 'Edwin. L. Steele Laboratories for Tumor Biology, Department of Radiation Oncology, \\ Massachusetts General Hospital and Harvard Medical School, Boston, MA 20114, USA \\ 2Department of General Surgery, Sir Run Run Shaw Hospital, Zhejiang University, \\ Hangzhou, China \\ ${ }^{3}$ Department of Anatomy, Tokyo Women Medical University, Tokyo, Japan \\ ${ }^{4}$ Department of Surgery, Keio University, Tokyo, Japan \\ Corresponding author: \\ Jiang Chen, MD, PhD \\ Edwin L. Steele Laboratories for Tumor \\ Biology, Department of Radiation \\ Oncology, Massachusetts General \\ Hospital, 100 Blossom St, Cox-734 \\ Boston, MA 02478, USA \\ Fax: 617-726-1962. \\ E-mail: JCHEN106@mgh.harvard.edu \\ or Shuji Kitahara, DDS, PhD \\ E-mail: skitahara@twmu.ac.jp,
}

\section{ABSTRACT}

The curative approach to advanced gastric cancer treatment is surgical resection. But the risk of relapse after resection in this disease remains high. To address this, multiple perioperative local and systemic therapies have been tested in patients with operable gastric adenocarcinomas over the last two decades. However, while the roles of neoadjuvant and adjuvant therapies have been evolving over the last two decades, many unanswered questions remain. Here, we first discuss the scientific rationale for these interventions. Next, we review the current progress, and offer a perspective on potential approaches that may be useful in the perioperative setting in advanced gastric cancer.

Key words: perioperative therapy, gastric cancer, adjuvant therapy, combination therapy, antiangiogenic therapy, immune checkpoint blockade

\section{INTRODUCTION}

Gastric cancer (GC) is the third most common cancer, with one million newly diagnosed patients per year, and the third most deadly cancer worldwide (1). The 5-year survival of patients with early GC is about $75 \%$, but at an advanced stage with extensive lymph node involvement, it drops to less than $30 \%$. Unfortunately, most cases of GC are diagnosed at advanced stage. Therefore, improving the efficacy of therapy for patients with advanced GC remains an key aspect for overall treatment outcomes of this disease (2).

The vast majority of GCs are adenocarcinomas, which can be further subdivided into intestinal and diffuse types according to the Lauren classification (3). Tumor microenvironmental factors such as hypoxia, angiogenesis, fibrosis, and inflammation are critical processes in the for local progression and distant metastasis of solids tumors including GC (4). These factors do so by creating an immune-suppressive tumor microenvironment characterized by hypoxia and immunosuppression. As a consequence, no existing single chemotherapy agent or combination regimen consistently leads to objective tumor shrinkage, and 
novel therapy approaches for GCs are desperately needed (4).

\section{Tumor vasculature as a target for $G C$}

In addition to genetic abnormalities, tumors develop a new blood vasculature through a process referred to as angiogenesis - which results in largely immature vessels (5-7). In malignant tumors, angiogenesis is often driven by the vascular endothelial growth factor (VEGF)/VEGF receptor VEGFR-2 pathway; the abnormal vessels resulting from overexpression of VEGF and other angiogenic factors are associated with an abnormal tumor microenvironment characterized by low tissue oxygenation (hypoxia) and immunosuppression (8). These characteristics of the microenvironment promote tumor progression as well as resistance to chemo-, radio-, and immune-basedtherapies. Much progress has been achieved in the last half of the century, since targeting tumor angiogenesis has been proposed as a strategy for cancer therapy in particular by targeting the VEGF pathway (9). The concept introduced by Dr. Rakesh K. Jain (Harvard University) posited that the appropriate dose of antiangiogenic treatment can lead to a normalization of the tumor vasculature, by reducing vascular permeability and interstitial fluid pressure; in turn, these changes will improve blood flow and perfusion (10). In this manner, a normalized tumor vasculature could enhance the delivery of anti-cancer drugs and of oxygen, important for radiation therapy, and promote anti-tumor immunity (11). Several preclinical and clinical studies supported this hypothesis (4). In metastatic GC, anti-VEGFR-2 antibody therapy is a standard of care alone or in combination with chemotherapy (see below). However, while antiangiogenic therapy has shown feasibility in the perioperative setting in several cancers, its rationale remains unclear given the lack of efficacy seen in large studies of anti-VEGF/VEGFR-2 antibodies with chemotherapy in colorectal and breast cancer patients (12-15).

\section{Promoting anti-tumor immunity as an approach for GC therapy}

Targeting immune evasion by malignant tumors has been a major unmet need in oncology. While the immune response to a specific antigen is recognized by major histocompatibility receptors, co-stimulatory and co-inhibitory molecules called immune checkpoints regulate the intensity of response. Immune checkpoints are physiologically expressed by normal for the maintenance of self-tolerance (16). In tumors, immune checkpoint molecules such as programmed cell death receptor (PD)-1, PD ligands (PD-L)-1 and -2 and cytotoxic T-lymphocyte-associated-protein (CTLA)-4 are often overexpressed and mediate immune evasion (17). Recently, monoclonal antibodies that block immune checkpoints such as PD-1/PD-L1 or CTLA-4 are transforming the treatment of patients with advanced cancers (18). So far, these drugs have profoundly changed the treatment of several advanced/metastatic cancers (for example melanoma or lung cancer). Integrating these new immunotherapies in the perioperative (neoadjuvant and/or adjuvant) therapy could potentially have an important impact for down-staging locally advanced tumors and increasing the durability treatment responses $(2,19)$. However, the determinants of this response are incompletely understood in GC. The tumor microenvironment is thought to play a key role in the response to immunotherapy with immune checkpoint inhibitors (4). The infiltrating immune cells such as cytotoxic T-lymphocyte in tumors may differ widely in density, composition, and clinical significance (20). Tumor vessels are a critical part of the immune microenvironment that can influence immunotherapy response and can be targeted using anti-angiogenic drugs $(4,21,22)$. Blood vascular and lymphatic endothelial cells may also play important roles in the trafficking of immune cells, controlling the microenvironment, and modulating the immune response. Improving access to the malignant tumor through vascular alterations induced by anti-angiogenic drugs may prove an effective combinatorial strategy with immunotherapy approaches and might be applicable to many tumor types, especially GC, where both anti-PD-1 and anti-VEGFR-2 antibodies are standard of care in metastatic disease (23). This knowledge may be important to devise new strategies to more substantially improve overall survival in GC patients, for example by combining anti-angiogenic agents with immunotherapy such as immune checkpoint inhibitors.

\section{Why target the immune checkpoints in gastric cancer?}

Anti-immune checkpoint therapy for GC is attractive for several reasons. The majority of GCs are associated with infectious agents, including the bacterium Helicobacter (H.) pylori and EBV (24). Thus, GCs may be highly immunogenic, especially when induced by Epstein Barr Virus (EBV) infection or when presenting with 'microsatellite instability' (MSI). Similarly, a high 
mutational burden may increase the likelihood of response to anti-immune checkpoint blockade (1). Indeed, immunotherapy has shown great promise to radically improve durability of responses and outcomes in certain advanced cancers, including in metastatic GC. However, in the majority of patients, therapy fails to elicit an appropriate anti-tumor immune response. In part, this treatment resistance may be mediated by the typically immunosuppressive GC microenvironment. Thus, much progress needs to be made in our understanding of determinants of immune evasion prior to integration of immunotherapy for neoadjuvant and/or adjuvant therapy in advanced GC.

\section{How does gastric cancer evade immunity?}

A previous report by the TCGA project reported that $15 \%$ of GCs showed amplified PD-L1 expression in EBV-positive GCs. Evaluation of mRNA revealed elevated expression of JAK2, PD-L1 and PD-L2 in the amplified cases (1). In addition, Lin et al. reported was significantly enriched in signatures related to T-cell activation, including CTLA-4 signaling in GC samples from non-Asian patients. Similarly, in non-Asian GCs showed significantly higher expression of T-cell markers and lower expression of the immunosuppressive T-regulatory (Treg) cell marker FoxP3 (25). Furthermore, in lymphocyte-rich GCs, the tumor stroma could serve as a "tertiary lymphoid tissue" (26). Multiple other immune-suppressive mechanisms have been also proposed. The stomach tissue is inherently is "tolerogenic" to prevent aberrant immunity in response to these potential antigens absorbed from epithelium $(27,28)$. On the other hand, GCs are inflammation-induced malignancies, because they often occur in a diseased stomach on the background of gastritis (29). The underlying chronic inflammation and viral infection create an immuno-suppressive environment in the stomach through the production of cytokines including interleukin (IL)-6, IL-11, tumor necrosis factor-alpha (TNF- $\alpha$ ), and transforming growth factor-beta (TGF- $\beta)(21,30)$. The infiltration of tumors by immuno-suppressive cells, e.g., Tregs and myeloidderived suppressor cells (MDSCs) is another important mechanism of immune evasion in cancer. Exhaustion of effector $T$ cells has also been reported as a mechanism of immune evasion in advanced cancer patients $(31,32)$.

\section{Anti-vascular therapy can modulate immune suppressive microenvironment}

Reactivation of immune response is a key to achieving efficacy for immunotherapy in GC. Growing evidence is showing that combining anti-angiogenic therapy with immunotherapy may in certain contexts improve immune response in solid cancers $(11,33)$. Several other studies have evaluated the change in immune response after anti-angiogenic therapy. For example, bevacizumab has shown to enhance dendritic cell (DC) proportion and function in solid cancer patients (34). In mouse models, it has been reported that anti-VEGF antibody can enhance the number and function of DC (35). Previously, Huang et al. reported the impact of dose titration for antiVEGFR2 antibody therapy on vascular normalization, reprogramming of the immune microenvironment and vaccine immunotherapy in a mouse model of breast cancer (36). This preclinical evidence suggests the potential benefit of titration of anti-VEGFR2 antibody therapy on immunotherapy in GC.

\section{Evolving role of targeting immune checkpoints in $G C$}

Anti-PD-1/PD-L1 therapy is the most advanced modality for immune checkpoint blockade across cancer types, including GC. PD-1 is a CD28 super family member that conveys co-inhibitory signals for $\mathrm{T}$ cell receptor (TCR) (37). PD-1 is expressed in CD8+ T cells, Tregs and MDSCs $(38,39)$. In addition, PD-1 mediates peripheral tolerance and autoimmunity. Chronic exposure to antigen may increase PD-1 expression in T cells, leading to anergy or cell exhaustion (40). Cancer cells can "hijack" PD-1 signaling by expressing PD-L1 or PD-L2 to activate PD-1 in tumor-infiltrating lymphocytes and evade immune surveillance (41). PD-1 and PD-L1 expression can be detected in clinical samples and significantly correlated with human GC stage (23), local recurrence rate and poor prognosis (1). All these data support the evolving role of immune checkpoint inhibition therapy in GC. Unfortunately, as discussed below, only a fraction of GC patients respond to this intervention, highlighting the need for patient selection for this therapy modality, as well as other targeted therapies. 


\section{Potential for using new classification methods for improving GC therapy}

Traditional staging systems based on tumor location or morphology have a proven utility in predicting prognosis of GC patients. These systems are also useful in stratifying patients according to prognostic variables in the setting of clinical trials, and allowing the exchange of information among researchers, and finally guiding the therapeutic approach $(42,43)$. Although surveillance protocols of patients at-risk to develop GC have significantly improved in this manner, the clinical outcome remains poor with a majority of patients presenting with advanced disease and not being candidates for surgery. Although several new classification systems have been proposed recently to adapt to tumor biological diversity and new complicated combination therapies, classification and biomarker screening for antibody-targeted therapy and immunotherapy are desperately needed for patient selection for implementation of new adjuvant and/or neoadjuvant therapies $(23,42)$.

\section{Role of chemotherapy in adjuvant setting in GC patients}

The role of perioperative chemotherapies has been proposed after the regimen of epirubicin, cisplatin and infused fluorouracil (FU) (ECF) showed a good response rate $(49-56 \%)$ in randomized trials for unresectable advanced gastric cancers $(44,45)$. From the early 2000 s, development of chemotherapy had been focused on its use as perioperative treatment. A key randomized trial of perioperative chemotherapy for patients with resectable gastro-esophageal cancer was reported in The New England Journal of Medicine (46). In this study, 3 cycles of both preoperative and postoperative ECF were planned. Although only $42 \%$ patients enrolled in the perioperative chemotherapy group could complete all treatment as per protocol, the result showed improvement of progression-free survival (PFS) (Hazard ratio $[\mathrm{HR}]=0.66)$ and 5 -year survival rate $(36 \%$ vs $23 \%, \mathrm{HR} ; 0.75)$.

\section{Role of chemoradiation in adjuvant setting in GC patients}

The benefit of adjuvant chemoradiotherapy (CRT) was reported by the Intergroup (INT)-0116 in a trial in GC patients performed two decades ago (47). Study enrolled 556 patients who were randomized to surgery followed by 1 cycle of $5-\mathrm{FU}$, followed by radiation concurrent with 5-FU administered for 4 days at the beginning of radiation and during the last 3 days of radiation versus surgery alone. Trial data demonstrated that patients enrolled in the CRT group had a medial superior overall survival (OS). Compared to the surgery alone group, GC patients who underwent postoperative CRT had a significantly lower rate of relapse, not only local but also regional. An updated analysis of the INT-0116 study showed a persistent PFS and OS benefit for adjuvant CRT (5-year OS $43 \%$ vs $28 \%$, $H R=1.32)$ (48). Despite these significant benefits in a randomized phase III trial, the role of adjuvant CRT remains controversial. In the Adjuvant CRT in Stomach Cancer (ARTIST) trial, 458 patients received extensive lymph node dissection (D2) surgery and were then randomized to receive adjuvant capecitabine plus cisplatin (XP) before and after CRT versus XP alone. Study data demonstrated that CRT did not increase OS, but improved relative disease-free survival (DFS) by $32 \%$ among 300 patients with positive lymph node involvement according to an unplanned subgroup analysis (49). Another RCT study compared the adjuvant CRT regimen used in the INT-0116 study to chemotherapy alone (without radiation). Compared to the chemotherapy alone group, the 5-year disease-free survival was significantly superior in the CRT group (50). However, the Chemoradiotherapy after Induction Chemotherapy in Cancer of the Stomach (CRITICS) trial showed no difference between CRT and chemotherapy alone (51). Currently, the ARTIST-II trial (NCT01761461) is ongoing and the goal is validating that CRT will benefit patients with positive lymph nodes over chemotherapy alone - based on the results of subgroup analysis in the ARTIST trial. Given the inconsistent results, and the potential impact of regional differences in surgical interventions, future studies need to address the role of CRT in adjuvant setting. Thus, while adjuvant cytotoxic therapy has become one of the standard therapeutic options for advanced GC after resection, there is no international consensus for adjuvant therapies in this setting.

In the US, the National Comprehensive Cancer Network (NCCN) clinical practice guidelines recommend CRT for advanced GS patients who have not received preoperative chemotherapy (52). In Europe, the United Kingdom Medical Research Council Adjuvant Gastric Infusional Chemotherapy (MAGIC trial) enrolled 503 patients with potentially resectable gastric, distal esophageal or esophagogastric junction adenocarcinomas who were randomized to surgery alone or chemotherapy with ECF. Adjuvant ECF chemotherapy improved median OS ( $\mathrm{HR}=0.75)$ and PFS 
$(H R=0.66)$ (46). However, the European Society for Medical Oncology (ESMO) clinical practice guidelines recommend perioperative chemotherapy for advanced GC preferentially (53). For patients who have undergone gastrectomy without preoperative therapy, postoperative CRT or chemotherapy are recommended.

In East Asia, S-1 chemotherapy demonstrated significant efficacy as adjuvant treatment for locally advanced GC patients who have undergone a D2 dissection (54). Based on these data, Japanese GC treatment guidelines recommend postoperative chemotherapy with S-1 monotherapy (55). Based on recently findings from Fuse et al. and the Capecitabine and Oxaliplatin Adjuvant Study in Stomach Cancer (CLASSIC) study, the XELOX (capecitabine plus oxaliplatin) regimen can be considered an adjuvant treatment option for GC patients who have undergone curative resection (5-year OS rate was $78 \%$ vs $69 \%$ with surgery alone, $\mathrm{HR}=0.66)(56)$.

In summary, although the outcomes for patients with locally advanced GC have improved substantially with the advent of with chemotherapy and CRT, future studies need to establish their respective roles and allow further optimization of adjuvant therapy.

\section{Role of chemotherapy in the neoadjuvant setting in GC patients}

A recent study by Al-Batran et al. reported promising data from a clinical trial of neoadjuvant chemotherapy in advanced GC patients including limited metastatic disease who underwent resection and showed a favorable survival. This trial (AIO-FLOT3) provided a rationale for further randomized clinical trials (57). The issue of neoadjuvant therapy is addressed in several ongoing clinical trials, such as Neoadjuvant FOLFIRINOX in the Treatment of Locally Advanced Gastric Cancer (NCT03825861 and Randomized Phase III Trial of Surgery Plus Neoadjuvant TS-1 and Cisplatin Compared With Surgery Alone for Type 4 and Large Type 3 Gastric Cancer: Japan Clinical Oncology Group Study (JCOG 0501) (NCT00252161).

\section{Role of chemoradiation in the neoadjuvant setting in GC patients}

Neoadjuvant chemoradiation has been proposed as an approach to improve RO resection rates and achievement of pathological complete responses ( $p C R s$ ) in advanced GC. However, several meta-analyses compared neoadjuvant CRT versus CT for adeno- carcinoma of gastroesophageal junction (GEJ), and found no difference in median OS despite a higher $\mathrm{pCR}$ rate and a reduced risk of locoregional recurrences for the CRT approach (58). Several phase II and phase III clinical trials are ongoing to prospectively address the role of CRT in neoadjuvant setting, including " $A$ Randomized, Multicenter, Open-label, Phase III Trial Comparing Neoadjuvant Chemoradiotherapy Versus Chemotherapy in Patients With Locally Advanced Gastric Adenocarcinoma (NCT01815853)" and "A Phase II Study of Neoadjuvant Chemotherapy With S1+ Oxaliplatin (SOX) Regimen Followed by Chemoradiation Concurrent With S-1 in Patients With Potentially Resectable Gastric Carcinoma (NCT02024217)".

\section{The rationale for molecularly targeted therapies, antiangiogenic therapies, and immunotherapies}

\section{Molecularly targeted therapy}

Molecularly targeted therapy has shown promise in advanced GC, as one of the examples of personalized therapy. Human epidermal growth factor receptor 2 (HER2) is a critical oncogenic driver in a subset of GC and GEJ cancers. Previous studies suggested that HER2 amplification and/or overexpression in these tumors leads to poor outcomes and a more aggressive phenotype. That provided rationale for the use of trastuzumab - an effective anti-HER2 blocking antibody in HER2-driven breast cancers - in combined with chemotherapy to treat HER2-positive advanced GC.

Indeed, the ToGA trial was the first clinical trial to confirm that trastuzumab plus chemotherapy can improve survival in advanced GC patients (59). This trial recruited 594 patients who were randomized to receive trastuzumab plus chemotherapy or chemotherapy alone. Compared to patients treated with chemotherapy alone, the median OS was more than 2 months longer in the patients treated with trastuzumab plus chemotherapy. Better yet, the risk of death was reduced by $26 \%$. Multiple phase II and phase III trials are now testing chemotherapy regimens combined with trastuzumab/pertuzumab (another antibody which prevents the HER2/HER3 dimer formation) in neoadjuvant/perioperative setting for HER2-positive advanced GC (e.g., NCT02205047, NCT NCT02581462, NCT03691454). In Japan, the JCOG1301 phase III study is currently underway to evaluate the efficacy of using trastuzumab in combination with S-1/cisplatin based on promising phase II data (60). 


\section{Antiangiogenic therapy}

As discussed above, tumor vessel formation and function are critical for cancer progression and response to treatment. Angiogenesis is a crucial aspect of tumorigenesis and progression of solid tumors such as GC. VEGF/VEGFR-2 pathway is a key mediator of pathologic and physiologic angiogenesis. This understanding has prompted trials of agents targeting the VEGF or VEGFR-2 in advanced GC.

The randomized phase III AVAGAST trial tested the efficacy of bevacizumab, a monoclonal anti-VEGF blocking antibody, with cisplatin and fluoropyrimidine versus chemotherapy alone in metastatic or unresectable advanced GC patients. The results demonstrated that bevacizumab plus chemotherapy can significantly improve median PFS but not OS (61). The REGARD trial evaluated an anti-VEGFR-2 blocking antibody (ramucirumab) as a second-line option for patients with metastatic disease unresponsive to first-line chemotherapy. Trial data showed that ramucirumab can significantly improve OS with these patients (62). This conclusion was further validated by other clinical trials. A phase 3 trial confirmed that combination of ramucirumab with paclitaxel chemotherapy significantly increased median OS compared with placebo plus paclitaxel, and thus could be regarded as a new standard second-line treatment for patients with advanced GC (63). Finally, the pan-VEGFR tyrosine kinase inhibitor apatinib demonstrated increased response rates, PFS and OS when combined with chemotherapy in advanced GC in a trial in Chinese patients (64).

Despite the proven efficacy of this approach, important challenges remain for the integration of antiangiogenic perioperative setting. Anti-VEGF agents did not prevent metastasis in other cancers and their role in neoadjuvant setting in advanced GC remains to be established.

\section{Immunotherapies}

As discussed above, some GCs could be relatively immunogenic. In these cancers, the immune response is suppressed by multiple mechanisms, and immune checkpoint blockade therapy may be an effective strategy to reactivate anti-tumor immunity. There are multiple clinical trials exploring the role of immune checkpoint blockade therapy (PD1/PD-L1, CTLA4, VISTA, etc.) in GC, including in advanced disease. Others are testing on tumor-specific $T$ lymphocytes, natural killer cells, dendritic cell-based vaccines and cytokine-induced killer (CIK) cells.
PD-1. A case report completed by Yu S et al, demonstrated that combination of concurrent PD-1 antibody immunotherapy, chemotherapy (S-1 plus oxaliplatin) and radiotherapy could be effective against patients with advanced GC (65). In ATTRACTION-2 clinical trial of the anti-PD-1 antibody nivolumab as a salvage therapy versus placebo group, immunotherapy demonstrated significant survival benefits in patients with advanced GC/GEJ cancer previously treated with chemotherapy (66). The efficacy of nivolumab in combination with postoperative chemotherapy will be tested, in the phase III study ATTRACTION-05 (NCT03006705). The anti-PD-1 antibody pembrolizumab is currently being tested in advanced GC in the phase III trial (KEYNOTE585 study) (67). This clinical trial is designed to evaluate the efficacy of pembrolizumab in combination with perioperative chemotherapy for patients with locally advanced GC and GEJ cancer. As a subgroup of $1 b$ KEYNOTE-012 study, Muro $\mathrm{K}$ et al. assessed the safety and activity of pembrolizumab in patients with PD-L1positive recurrent or metastatic GC and GEJ cancers (68). The study results showed that pembrolizumab had a tolerable toxicity profile and promising antitumor activity. Further evaluation of the safety and efficacy of pembrolizumab in previously treated GC and GEJ cancers, in the phase II clinical trial (KEYNOTE-059 study) showed that pembrolizumab demonstrated promising activity in these patients who had previously treatment (69). Durable responses were observed not only in patients with PD-L1-positive, but also in patients with PD-L1-negative tumors. In a randomized, open-label, controlled, phase III trial, pembrolizumab did not show increase survival despite showing a more favorable safety profile compare to paclitaxel (70). Toripalimab is a humanized IgG4 monoclonal antibody against PD-1 and has demonstrated a manageable safety profile and promising antitumor activity in advanced GC patients, especially in combination with XELOX (71). There are several ongoing clinical trials testing immunotherapy combined with chemotherapy in neoadjuvant setting, e.g., a phase II clinical study of neoadjuvant therapy for resectable locally advanced GC with PD-1 antibody or in combination with apatinib \pm S1 (NCT03878472); a phase II study of PD-1 inhibitor combined with FOLFOX neoadjuvant therapy for resectable GC and GEJ Adenocarcinoma (NCT03939962); SHR-1210 combined with trastuzumab, oxaliplatin and capecitabine for neoadjuvant therapy of GC/GEJ adenocarcinoma (NCT03950271).

Multiple other trials of anti-PD-1 antibodies are ongoing. 


\section{$P D-L 1$}

With an increase in the use of adjuvant CRT for locally advanced GC after surgical resection, researchers also found a relationship between radiotherapy and increased PD-L1 expression. Zhang et al. observed that the expression of PD-L1 was increased in irradiated cancer cell lines in vitro (72). They hypothesized that PD-L1 expressing tumor cells might be selected during radiotherapy to resist anti-tumor immune responses. Moreover, Yu et al. reported that following neoadjuvant chemotherapy, the expression levels of PD-L1, PD-1 and TIM-3 were significantly increased in GC patients (73). Based on this premise, several clinical trials are investigating the role of antiPD-L1 immunotherapy in advanced cancer, including in perioperative setting (74).

CTLA-4. The anti-CTLA-4 antibody tremelimumab was one of the first immune checkpoint inhibitors investigated in patients with GC/GEJ cancers. In a phase II study, Ralph et al. investigated tremelimumab in 18 patients and reported that approximately one-third of patients were alive at one year (75). The anti-CTLA-4 antibody Ipilimumab was also tested in a randomized phase II trial, but the study was stopped early due to lack of clinical efficiency. While the role of targeting CTLA-4 alone remains unclear, an ongoing phase I/II study is investigating the role of dual PD-1/CTALA-4 blockade (nivolumab/ipilimumab) with CRT in patients with resectable GC (NCT03776487). Janjigian et al. reported that nivolumab and nivolumab plus ipilimumab demonstrated clinically meaningful antitumor activity, durable responses, encouraging long-term OS, and a manageable safety profile in patients with chemotherapy-refractory esophagogastric cancer (76). Phase III studies evaluating nivolumab or nivolumab plus ipilimumab in earlier lines of therapy for esophagogastric cancers are also underway.

Other immunotherapy approaches. Other immunotherapy approaches include tumor-specific T lymphocyte, cytokine-induced killer (CIK) cell or natural killer cell therapy. In a cohort study, Kuai et al. reported that in vitro activated tumor-specific $T$ lymphocytes can prolong the survival of patients with advanced GC. In this study, in vitro activated tumor-specific T lymphocytes immunotherapy plus chemotherapy after surgery prolonged the median survival of advanced GC patients by 9.8 months compared with chemotherapy alone (77). Similarly, CIK cell have shown promising activity. Chen et al. showed that adjuvant chemotherapy combined with autologous CIK therapy could improve outcome for GC patients after D2 gastrectomy compared to chemotherapy alone. Study data showed that patients in the CIK group had longer DFS and OS than patients in the control group (DFS 41 vs. 32.0 months and OS 45 vs. 44 months) (78). Finally, in a phase I clinical trial, Ishikawa et al. provided initial evidence of anti-tumor activity after transfer of expanded NK cells in combination with IgG1 antibody in patients with GC or colorectal cancer that have received previous therapy (79).

Despite this advance in our understanding, assessing immunotherapy responses remains challenging as tumors may demonstrate atypical responses, adverse reactions and pseudo-progression. Atypical therapy response is crucial for physicians to understand. Based on RECIST 1.1, the RECIST working group modified the response criteria, and IRECIST can now provide guidance for response assessment of oncologic patients treated with immunotherapy (80). The use of IRECIST should be further validated for making therapy decisions, but it may potentially optimize therapy outcomes.

\section{CONCLUSION}

Radical gastrectomy with lymph node dissection is a standard treatment for advanced GC. Cytotoxic therapies are effective in perioperative setting, and ongoing studies may establish the optimal regimens. For patients who do not receive preoperative chemotherapy, postoperative chemoradiation is usually recommended. Future directions include incorporation of targeted therapies - which have shown efficacy in metastatic setting - earlier in the disease management. These therapies include anti-angiogenic, anti-HER2 and immune checkpoint blockade. In addition, multimodal combination therapies might be feasible to address treatment resistance. Despite while many challenges remain, including the high costs and potential serious toxicities associated with these therapies, compelling evidence supports the potential efficacy of such combinations. However, mechanism- and biomarkerbased approaches are needed to guide study and treatment design. Research in this area will lay the groundwork for a new paradigm of combination immunotherapy as a new adjuvant and/or neoadjuvant therapy that could rapidly impact the management of advanced GC.

\section{Conflict of interest}

The authors report no conflicts of interest in this work. 


\section{REFERENCES}

1. Cancer Genome Atlas Research N. Comprehensive molecular characterization of gastric adenocarcinoma. Nature 2014;513:202-9.

2. Hu SB, Liu CH, Wang X, Dong YW, Zhao L, Liu HF, et al. Pathological evaluation of neoadjuvant chemotherapy in advanced gastric cancer. World J Surg Oncol 2019;17(1):3.

3. Hu B, El Hajj N, Sittler S, Lammert N, Barnes R, Meloni-Ehrig A. Gastric cancer: Classification, histology and application of molecular pathology. J Gastrointest Oncol 2012;3(3):251-61.

4. Park do J, Thomas NJ, Yoon C, Yoon SS. Vascular endothelial growth factor a inhibition in gastric cancer. Gastric Cancer 2015; 18(1):33-42.

5. Kitahara S, Morikawa S, Shimizu K, Abe H, Ezaki T. Alteration of angiogenic patterns on B16BL6 melanoma development promoted in Matrigel. Med Mol Morphol 2010;43(1):26-36.

6. Baluk P, Morikawa S, Haskell A, Mancuso M, McDonald DM. Abnormalities of basement membrane on blood vessels and endothelial sprouts in tumors. Am J Pathol. 2003;163(5):1801-15.

7. Goel S, Duda DG, Xu L, Munn LL, Boucher Y, Fukumura D, et al. Normalization of the vasculature for treatment of cancer and other diseases. Physiol Rev. 2011;91(3): 1071-121.

8. Jain RK. Normalization of Tumor Vasculature: An Emerging Concept in Antiangiogenic Therapy. Science 2005;307:58-62.

9. Folkman J. Tumor angiogenesis: therapeutic implications. N Engl J Med 1971;285:1182-6.

10. Jain RK. Normalizing tumor microenvironment to treat cancer: bench to bedside to biomarkers. J Clin Oncol 2013;31:2205-18.

11. Huang Y, Stylianopoulos T, Duda DG, Fukumura D, Jain RK. Benefits of vascular normalization are dose and time dependent--letter. Cancer Res 2013;73(23):7144-6.

12. Burstein HJ, Chen YH, Parker LM, Savoie J, Younger J, Kuter I, et al. VEGF as a marker for outcome among advanced breast cancer patients receiving anti-VEGF therapy with bevacizumab and vinorelbine chemotherapy. Clin Cancer Res 2008;14(23):7871-7.

13. Duda DG, Willett CG, Ancukiewicz M, di Tomaso E, Shah M, Czito BG, et al. Plasma soluble VEGFR-1 is a potential dual biomarker of response and toxicity for bevacizumab with chemoradiation in locally advanced rectal cancer. Oncologist 2010;15(6):577-83.

14. Rahbari NN, Kedrin D, Incio J, Liu H, Ho WW, Nia HT, et al. AntiVEGF therapy induces ECM remodeling and mechanical barriers to therapy in colorectal cancer liver metastases. Sci Transl Med 2016; 8(360):360ra135.

15. Terme M, Pernot S, Marcheteau E, Sandoval F, Benhamouda N, Colussi 0, et al. VEGFA-VEGFR pathway blockade inhibits tumorinduced regulatory T-cell proliferation in colorectal cancer. Cancer Res 2013;73(2):539-49.

16. Okazaki T, Chikuma S, Iwai Y, Fagarasan S, Honjo T. A rheostat for immune responses: the unique properties of PD-1 and their advantages for clinical application. Nat Immunol 2013;14(12):1212-8.

17. Xu C, Fillmore CM, Koyama S, Wu H, Zhao Y, Chen Z et al. Loss of Lkb1 and Pten leads to lung squamous cell carcinoma with elevated PD-L1 expression. Cancer Cell 2014;25(5):590-604.

18. Hodi FS, O'Day SJ, McDermott DF, Weber RW, Sosman JA, Haanen $\mathrm{JB}$, et al. Improved survival with ipilimumab in patients with metastatic melanoma. N Engl J Med 2010;363(8):711-23.

19. Schirren R, Reim D, Novotny AR. Adjuvant and/or neoadjuvant therapy for gastric cancer? A perspective review. Ther Adv Med Oncol 2015;7:39-48.

20. Chen $\mathrm{Y}$, Ramjiawan RR, Reiberger $\mathrm{T}, \mathrm{Ng} \mathrm{MR}$, Hato T, Huang $\mathrm{Y}$, et al. CXCR4 inhibition in tumor microenvironment facilitates antiprogrammed death receptor-1 immunotherapy in sorafenib-treated hepatocellular carcinoma in mice. Hepatology 2015;61(5):1591602.

21. Zhang S, Desrosiers J, Aponte-Pieras JR, DaSilva K, Fast LD, Terry
F, et al. Human immune responses to H. pylori HLA Class II epitopes identified by immuno-informatic methods. PLoS One 2014;9(4): e94974.

22. Yoshii $\mathrm{A}$, Kitahara $\mathrm{S}$, Ueta $\mathrm{H}$, Matsuno K, Ezaki T. Role of uterine contraction in regeneration of the murine postpartum endometrium. Biol Reprod 2014;91(2):32.

23. Setia N, Agoston AT, Han HS, Mullen JT, Duda DG, Clark JW, et al. A protein and mRNA expression-based classification of gastric cancer. Mod Pathol 2016;29(7):772-84.

24. Oh JK, Weiderpass E. Infection and Cancer: Global Distribution and Burden of Diseases. Ann Glob Health 2014;80:384-392.

25. Lin SJ, Gagnon-Bartsch JA, Tan IB, Earle S, Ruff L, Pettinger K, et al. Signatures of tumour immunity distinguish Asian and non-Asian gastric adenocarcinomas. Gut. 2015;64(11):1721-31.

26. Aloisi F, Pujol-Borrell R. Lymphoid neogenesis in chronic inflammatory diseases. Nat Rev Immunol. 2006;6:205-17.

27. Pelaseyed T, Bergstrom JH, Gustafsson JK, Ermund A, George M H Birchenough, André Schütte, et al. The mucus and mucins of the goblet cells and enterocytes provide the first defense line of the gastrointestinal tract and interact with the immune system. Immunol Rev. 2014;260(1):8-20.

28. Myint ZW, Goel G. Role of modern immunotherapy in gastrointestinal malignancies: a review of current clinical progress. J Hematol Oncol. 2017;10:86.

29. Yoon H, Kim N. Diagnosis and management of high risk group for gastric cancer. Gut Liver. 2015;9:5-17.

30. Putoczki TL, Thiem S, Loving A, Busuttil RA, Wilson NJ, Ziegler PK, et al. Interleukin-11 is the dominant IL-6 family cytokine during gastrointestinal tumorigenesis and can be targeted therapeutically. Cancer Cell. 2013(2);24:257-71.

31. Hoechst B, Voigtlaender T, Ormandy L, Gamrekelashvili J, Zhao F, Wedemeyer $\mathrm{H}$, et al. Myeloid derived suppressor cells inhibit natural killer cells in patients with hepatocellular carcinoma via the NKp30 receptor. Hepatology. 2009;50(3):799-807.

32. Gabitass RF, Annels NE, Stocken DD, Pandha HA, Middleton GW. Elevated myeloid-derived suppressor cells in pancreatic, esophageal and gastric cancer are an independent prognostic factor and are associated with significant elevation of the Th2 cytokine interleukin-13. Cancer Immunol Immunother. 2011;60(10):1419-30.

33. Zou Y, Meng J, Chen W, Liu J, Li X, Li W, et al. Modulation of phenotypic and functional maturation of murine dendritic cells (DCs) by purified Achyranthes bidentata polysaccharide (ABP). Int Immunopharmacol. 2011;11(8):1103-8.

34. Osada T, Chong G, Tansik R, Hong T, Spector N, Kumar R, et al. The effect of anti-VEGF therapy on immature myeloid cell and dendritic cells in cancer patients. Cancer Immunol Immunother 2008;57(8):1115-24.

35. Gabrilovich DI, Ishida T, Nadaf S, Ohm JE, Carbone DP. Antibodies to vascular endothelial growth factor enhance the efficacy of cancer immunotherapy by improving endogenous dendritic cell function. Clin Cancer Res. 1999;5(10):2963-70.

36. Huang Y, Yuan J, Righi E, Kamoun WS, Ancukiewicz M, Nezivar J, et al. Vascular normalizing doses of antiangiogenic treatment reprogram the immunosuppressive tumor microenvironment and enhance immunotherapy. Proc Natl Acad Sci U S A. 2012;109(43):17561-6.

37. Freeman GJ, Long AJ, Iwai Y, Bourque K, Chernova T, Nishimura H, et al. Engagement of the PD-1 immunoinhibitory receptor by a novel B7 family member leads to negative regulation of lymphocyte activation. J Exp Med 2000;192(7):1027-34.

38. Francisco LM, Salinas VH, Brown KE, Vanguri VK, Freeman GJ, Kuchroo VK, et al. PD-L1 regulates the development, maintenance, and function of induced regulatory $\mathrm{T}$ cells. J Exp Med 2009; 206(13):3015-29.

39. Nikolova M, Lelievre JD, Carriere M, Bensussan A, Lévy Y. 
Regulatory $\mathrm{T}$ cells differentially modulate the maturation and apoptosis of human CD8+ T-cell subsets. Blood. 2009;113(19): 4556-65.

40. Barber DL, Wherry EJ, Masopust D, Zhu B, Allison JP, Sharpe AH, et al. Restoring function in exhausted CD8 T cells during chronic viral infection. Nature 2006;439(7077):682-7.

41. Iwai $Y$, Ishida M, Tanaka $Y$, Okazaki $T$, Honjo $T$, Minato N. Involvement of PD-L1 on tumor cells in the escape from host immune system and tumor immunotherapy by PD-L1 blockade. Proc Natl Acad Sci U S A. 2002;99(19):12293-7.

42. Sitarz R, Skierucha M, Mielko J, Offerhaus GJA, Maciejewski R, Polkowski WP. Gastric cancer: epidemiology, prevention, classification, and treatment. Cancer Manag Res 2018;10:239-248.

43. Park DJ, Thomas NJ, Yoon C, Yoon SS. Vascular Endothelial Growth Factor A Inhibition in Gastric Cancer. Gastric Cancer. 2015;18(1) 33-42.

44. Webb A, Cunningham D, Scarffe JH, Harper P, Norman A, Joffe JK, et al. Randomized trial comparing epirubicin, cisplatin, and fluorouracil versus fluorouracil, doxorubicin, and methotrexate in advanced esophagogastric cancer. J Clin Oncol. 1997;15(1):261-267.

45. Ross $P$, Nicolson M, Cunningham D, Valle J, Seymour M, Harper P, et al. Prospective randomized trial comparing mitomycin, cisplatin, and protracted venous-infusion fluorouracil (PVI 5-FU) with epirubicin, cisplatin, and PVI 5-FU in advanced esophagogastric cancer. J Clin Oncol. 2002;20(8):1996-2004.

46. Cunningham D, Allum WH, Stenning SP, Thompson JN, Van de Velde CJ H, Nicolson M, et al. Perioperative chemotherapy versus surgery alone for resectable gastroesophageal cancer. N Engl J Med 2006;355(1):11-20

47. Macdonald JS, Smalley SR, Benedetti J, Hundahl SA, Estes NC, Stemmermann GN, et al. Chemoradiotherapy after surgery compared with surgery alone for adenocarcinoma of the stomach or gastroesophageal junction. N Engl J Med. 2001;345(10):725-30.

48. Smalley SR, Benedetti JK, Haller DG, Hundahl SA, Estes NC, Ajani JA, et al. Updated analysis of SWOG-directed intergroup study 0116 a phase III trial of adjuvant radiochemotherapy versus observation after curative gastric cancer resection. J Clin Oncol. 2012;30(19): 2327-33.

49. Lee J, Lim DH, Kim S, Park SH, Park JO, Park YS, et al. Phase III trial comparing capecitabine plus cisplatin versus capecitabine plus cisplatin with concurrent capecitabine radiotherapy in completely resected gastric cancer with D2 lymph node dissection: the ARTIST trial. J Clin Oncol. 2012; 30(3):268-73.

50. Zhu WG, Xua DF, Pu J, Zong C-d, Li T, Tao G-Z, et al. A randomized, controlled, multicenter study comparing intensity-modulated radiotherapy plus concurrent chemotherapy with chemotherapy alone in gastric cancer patients with D2 resection. Radiother Oncol 2012;104(3):361-6.

51. Claassen YHM, Hartgrink HH, de Steur WO, Dikken JL, van Sandick JW, van Grieken NCT, et al. Impact of upfront randomization for postoperative treatment on quality of surgery in the CRITICS gastric cancer trial. Gastric Cancer 2019;22(2):369-376.

52. Dikken JL, Jansen EP, Cats A, Bakker B, Hartgrink HH, MeershoekKlein Kranenbarg E, et al. Impact of the extent of surgery and postoperative chemoradiotherapy on recurrence patterns in gastric cancer. J Clin Oncol. 2010;28(14):2430-6.

53. Cunningham D, Starling N, Rao S, Iveson T, Nicolson M, Coxon F, et al. Capecitabine and oxaliplatin for advanced esophagogastric cancer. N Engl J Med 2008;358(1):36-46.

54. Sakuramoto S, Sasako M, Yamaguchi T, Kinoshita T, Fujii M, Nashimoto A, et al. Adjuvant chemotherapy for gastric cancer with S-1, an oral fluoropyrimidine. N Engl J Med 2007;357(18):1810-20.

55. Shitara K, Chin K, Yoshikawa T, Katai H, Terashima M, Ito S, et al Phase II study of adjuvant chemotherapy of S-1 plus oxaliplatin for patients with stage III gastric cancer after D2 gastrectomy. Gastric
Cancer 2017;20(1):175-181.

56. Fuse N, Bando H, Chin K, Ito S, Yoshikawa T, Tsuburaya A, et al. Adjuvant capecitabine plus oxaliplatin after D2 gastrectomy in Japanese patients with gastric cancer: a phase II study. Gastric Cancer 2017;20(2):332-340.

57. Al-Batran SE, Homann N, Pauligk C, Illerhaus G, Martens UM, StoehImacher J, et al. Effect of Neoadjuvant Chemotherapy Followed by Surgical Resection on Survival in Patients With Limited Metastatic Gastric or Gastroesophageal Junction Cancer: The AI0-FLOT3 Trial. JAMA Oncol 2017;3(9):1237-1244.

58. Petrelli F, Ghidini M, Barni S, Sgroi G, Passalacqua R, Tomasello G et al. Neoadjuvant chemoradiotherapy or chemotherapy for gastroesophageal junction adenocarcinoma: A systematic review and meta-analysis. Gastric Cancer 2019; 22(2):245-254.

59. Bang YJ, Van Cutsem E, Feyereislova A, Chung HC, Shen L, Sawaki A, et al. Trastuzumab in combination with chemotherapy versus chemotherapy alone for treatment of HER2-positive advanced gastric or gastro-oesophageal junction cancer (ToGA): a phase 3, open-label, randomised controlled trial. Lancet 2010;376(9742): 687-97.

60. Kataoka K, Tokunaga M, Mizusawa J, Machida N, Katayama H, Shitara K, et al. A randomized Phase II trial of systemic chemotherapy with and without trastuzumab followed by surgery in HER2positive advanced gastric or esophagogastric junction adenocarcinoma with extensive lymph node metastasis: Japan Clinical Oncology Group study JCOG1301 (Trigger Study). Jpn J Clin Oncol 2015;45(11):1082-6.

61. Ohtsu A, Shah MA, Van Cutsem E, Rha SY, Sawaki A, Park SR, et al. Bevacizumab in combination with chemotherapy as first-line therapy in advanced gastric cancer: a randomized, double-blind, placebocontrolled phase III study. J Clin Oncol 2011;29(30):3968-76.

62. Fuchs CS, Tomasek J, Yong CJ, Dumitru F, Passalacqua R, Goswami C, et al. Ramucirumab monotherapy for previously treated advanced gastric or gastro-oesophageal junction adenocarcinoma (REGARD): an international, randomised, multicentre, placebo-controlled, phase 3 trial. Lancet 2014;383(9911):31-39.

63. Wilke H, Muro K, Van Cutsem E, Oh S-C, Bodoky G, Shimada Y, et al. Ramucirumab plus paclitaxel versus placebo plus paclitaxel in patients with previously treated advanced gastric or gastrooesophageal junction adenocarcinoma (RAINBOW): a double-blind, randomised phase 3 trial. Lancet Oncol 2014;15(11):1224-35.

64. Hsieh HL, Tsai MM. Tumor progression-dependent angiogenesis in gastric cancer and its potential application. World J Gastrointest Oncol 2019:11:686-704.

65. Yu S, Cai L, Lin F, et al. Durable response after combination of concurrent chemoradiotherapy and anti-PD-1 therapy in HER2-negative advanced gastric adenocarcinoma: a case report. Onco Targets Ther 2019:12:7691-7698

66. Kang YK, Boku N, Satoh T, Ryu M-H, Chao Y, Kato K, et al. Nivolumab in patients with advanced gastric or gastro-oesophageal junction cancer refractory to, or intolerant of, at least two previous chemotherapy regimens (ONO-4538-12, ATTRACTION-2): a randomised, double-blind, placebo-controlled, phase 3 trial. Lancet 2017:390(10111):2461-2471.

67. Stewart C, Chao J, Chen YJ, Lin J, Sullivan MJ, Melstrom L, et al. Multimodality management of locally advanced gastric cancer - the timing and extent of surgery. Transl Gastroenterol Hepatol 2019;4:42 eCollection 2019

68. Muro K, Chung HC, Shankaran V, Geva R, Catenacci D, Gupta S, et al. Pembrolizumab for patients with PD-L1-positive advanced gastric cancer (KEYNOTE-012): a multicentre, open-label, phase 1b trial. Lancet Oncol 2016(6);17:717-726.

69. Fuchs CS, Doi T, Jang RW, Muro K, Satoh T, Machado M, et al. Safety and efficacy of pembrolizumab monotherapy in patients with previously treated advanced gastric and gastroesophageal junction 
cancer: phase 2 clinical KEYNOTE-059 Trial. JAMA Oncol 2018;4(5): e180013.

70. Shitara K, Ozguroglu M, Bang YJ, Di Bartolomeo M, Mandalà M, Ryu $\mathrm{M}-\mathrm{H}$, et al. Pembrolizumab versus paclitaxel for previously treated, advanced gastric or gastro-oesophageal junction cancer (KEYNOTE-061): a randomised, open-label, controlled, phase 3 trial. Lancet 2018;392(10142):123-133.

71. Wang F, Wei XL, Wang FH, Xu N, Shen L, Dai GH, et al. Safety, efficacy and tumor mutational burden as a biomarker of overall survival benefit in chemo-refractory gastric cancer treated with toripalimab, a PD-1 antibody in phase $\mathrm{Ib} / \mathrm{ll}$ clinical trial NCT02915432. Ann Oncol 2019;30(9):1479-1486.

72. Zhang W, Pang Q, Zhang X, Yan C, Wang Q, Yang J, et al. Programmed death-ligand 1 is prognostic factor in esophageal squamous cell carcinoma and is associated with epidermal growth factor receptor. Cancer Sci 2017;108(4):590-597.

73. Yu Y, Ma X, Zhang Y, Zhang Y, Ying J, Zhang W, et al. Changes in Expression of Multiple Checkpoint Molecules and Infiltration of Tumor Immune Cells after Neoadjuvant Chemotherapy in Gastric Cancer. J Cancer 2019;10(12):2754-2763.

74. Sato H, Suzuki Y, Yoshimoto Y, Noda S-E, Murata K, Takakusagi Y, et al. An abscopal effect in a case of concomitant treatment of locally and peritoneally recurrent gastric cancer using adoptive T-cell immunotherapy and radiotherapy. Clin Case Rep 2017;5(4):
380-384.

75. Ralph C, Elkord E, Burt DJ, O'Dwyer JF, Austin EB, Stern PL, et al. Modulation of lymphocyte regulation for cancer therapy: a phase II trial of tremelimumab in advanced gastric and esophageal adenocarcinoma. Clin Cancer Res 2010;16(5):1662-72.

76. Janjigian YY, Bendell J, Calvo E, Kim JW, Ascierto PA, Sharma P, et al. CheckMate-032 study: efficacy and safety of nivolumab and nivolumab plus ipilimumab in patients with metastatic esophagogastric cancer. J Clin Oncol 2018;36(28):2836-2844.

77. Kuai J, Yang F, Li GJ, Fang X-J, Gao B-Q. In vitro-activated tumorspecific $T$ lymphocytes prolong the survival of patients with advanced gastric cancer: a retrospective cohort study. Onco Targets Ther 2016;9:3763-70.

78. Chen Y, Guo ZQ, Shi CM, Zhou Z-F, Ye Y-B, Chen Q. Efficacy of adjuvant chemotherapy combined with immunotherapy with cytokine-induced killer cells for gastric cancer after d2 gastrectomy. Int J Clin Exp Med 2015;8(5):7728-36.

79. Ishikawa T, Okayama T, Sakamoto N, Ideno M, Oka K, Enoki T, et al. Phase I clinical trial of adoptive transfer of expanded natural killer cells in combination with IgG1 antibody in patients with gastric or colorectal cancer. Int J Cancer 2018;142(12):2599-2609.

80. Seymour L, Bogaerts J, Perrone A, Ford R, Schwartz LH, Mandrekar $S$, et al. iRECIST: guidelines for response criteria for use in trials testing immunotherapeutics. Lancet Oncol. 2017;18(3): e143-e152. 